\title{
Early prophylactic anticoagulation for portal vein system thrombosis after splenectomy: A systematic review and meta-analysis
}

\author{
NING ZHANG ${ }^{1 *}$, YINGMIN YAO $^{2 *}$, WANLI XUE ${ }^{3}$ and SHENGLI $\mathrm{WU}^{2}$ \\ Departments of ${ }^{1}$ Laboratory Medicine and ${ }^{2}$ Hepatobiliary Surgery, The First Affiliated Hospital of Xi'an \\ Jiaotong University, Xi'an; ${ }^{3}$ Department of Public Health, \\ Xi'an Jiaotong University School of Medicine, Xi'an, Shaanxi 710061, P.R. China
}

Received May 20, 2016; Accepted August 25, 2016

DOI: $10.3892 /$ br.2016.755

\begin{abstract}
A systematic review and meta-analysis were conducted to evaluate the efficacy and safety of early prophylactic anticoagulation for the prevention of portal vein system thrombosis (PVST) after splenectomy. A systematic search of the PubMed, EMBASE, Springer and Cochrane Library databases was performed to identify studies comparing the outcomes in patients receiving or not receiving regular prophylactic anticoagulation after splenectomy. The quality of the included studies was assessed using the Jadad Score and the Newcastle-Ottawa Scale. Heterogeneity was evaluated using the $\chi^{2}$ and $I^{2}$ tests. The parameters that were analyzed included the incidence of PVST and anticoagulation-associated complications. A total of seven studies qualified for the review, involving 383 and 283 patients receiving or not receiving regular prophylactic anticoagulation, respectively. The incidence of PVST was significantly reduced with an odds ratio (OR) of 0.31 [95\% confidence interval (CI), 0.21-0.46; $\mathrm{P}<0.00001]$ in the regular prophylactic anticoagulation group compared with the control group. No difference in the incidence of anticoagulation-associated complications was identified between the two groups $(\mathrm{OR}=0.60,95 \% \mathrm{CI}$, 0.23-1.56; $\mathrm{P}=0.30$ ). Early prophylactic anticoagulation was associated with a reduced incidence of PVST, although it was not associated with the incidence of anticoagulation-associated complications. These results indicate that prophylactic anticoagulation could be safely administered after splenectomy, even to cirrhotic patients.
\end{abstract}

Correspondence to: Dr Shengli Wu, Department of Hepatobiliary Surgery, The First Affiliated Hospital of Xi'an Jiaotong University, 277 Yanta West Road, Xi'an, Shaanxi 710061, P.R. China E-mail: victorywu2000@163.com

${ }^{*}$ Contributed equally

Key words: anticoagulation, portal vein system thrombosis, splenectomy, meta-analysis

\section{Introduction}

Portal vein system thrombosis (PVST) refers to the blood clots in portal vein, splenic and superior mesenteric veins or intrahepatic portal vein branches, as they form an interactive vascular system without valves (1). The clinical manifestations of PVST include asymptomatic to symptomatic fever, abdominal pain, nausea, vomiting and ileus (2). If not treated appropriately and in a timely fashion, PVST is potentially fatal by further increasing portal vein pressure and deteriorating liver function, which may increase the risk of upper gastrointestinal bleeding, hepatic coma or even fatal intestinal necrosis (3). With the introduction of advanced image devices, increasing numbers of studies have shown that the incidence of PVST secondary to splenectomy is significantly higher than previously reported (4). The majority of previous studies of PVST have predominantly focused on splenectomy to treat hematologic and metabolic disorders (5-7). A previous study found that cirrhotic patients also have a high risk of developing PVST after splenectomy, despite thrombocytopenia and a prolonged prothrombin time (8). Therefore, early prevention of PVST is considered to be important for avoiding adverse consequence in these patients. However, the role of early prophylactic anticoagulation in preventing PVST remains controversial, due to concerns regarding the risk of inducing bleeding, particularly in cirrhotic patients (9). By contrast, previous studies demonstrated that both pro- and anticoagulation elements were concomitantly reduced in liver cirrhosis, and an intricate balance of coagulation was thereby maintained $(10,11)$. Under such circumstances, the occurrence of bleeding in cirrhotic patients was primarily due to the severity of portal pressure, endothelial dysfunction and bacterial infections, but not the disturbed hemostasis (12). Accordingly, the prophylactic application of anticoagulation might be theoretically feasible for patients subsequent to splenectomy, including cirrhotic patients. There are previous studies that have identified that prophylactic anticoagulation therapy effectively prevents PVST after splenectomy. However, no standard regimen for PSVT prophylaxis has been developed and, to the best of our knowledge, there have been no systematic evaluations of the efficacy and safety of 
early prophylactic anticoagulation for the prevention of PVST following splenectomy.

Therefore, a systematic review and meta-analysis of the available studies was conducted. The impact of prophylactic anticoagulation on rates of PVST and anticoagulation-associated complications following splenectomy was assessed. These data may enable clinicians to establish effective methods to avoid this potential, lethal complication.

\section{Materials and methods}

Data sources and searches. A search of the PubMed (http://www.ncbi.nlm.nih.gov/pubmed/),EMBASE(www.embase .com), Springer (http://link.springer.com/), and Cochrane Library databases (http://www.cochranelibrary.com/) was performed with the following search terms as free-text terms, as well as MeSH terms: 'Portal vein thrombosis', 'portal venous thrombosis', 'splenectomy', 'anticoagulation' and 'anticoagulant'. The search was performed on March 2016. A manual search of the references of the relevant publications was also performed. No language restrictions were imposed.

Selection criteria. Studies reporting the outcome of prophylactic anticoagulation for the prevention of PVST subsequent to splenectomy were potentially eligible. The exclusion criteria were as follows: i) Duplicates; ii) review articles; iii) Case reports; iv) animal studies; v) studies in which PVST developed in malignant patients; and vi) studies not associated with the topics of interest.

Data extraction. The data extracted include: The first author, publication year, institution, study design, study period, study population, type of surgery, number of patients receiving or not receiving prophylactic anticoagulation, anticoagulation regimen (type of anticoagulants, dose of anticoagulants and duration of treatment), number of patients developing or not developing PVST and number of patients developing complications associated with anticoagulation.

Quality assessment. Two investigators independently read the titles and abstracts of potential studies, then read the full texts to identify eligible studies. The methodological quality of each randomized controlled trial (RCT) was assessed according to the Jadad score (13), including the following there aspects: Randomization, double blinding, and withdrawals and dropouts. Total scores of $0-2$ were considered low quality, whereas studies with total scores $\geq 3$ were defined as high quality. For each non-randomized study, the Newcastle-Ottawa Scale (NOS) (14) was used for quality assessment. A star system was used in which each study is judged on three broad perspectives: Selection, comparability and exposure for case-control studies. Studies with total stars $\geq 7$ were defined as high quality, 4-6 as medium and $\leq 4$ as low for quality (15).

Statistical analysis. The meta-analysis was performed using the statistical software RevMan 5.1 (The Cochrane Collaboration, http://tech.cochrane.org/revman). Pooled odds ratios (ORs) with 95\% confidence interval (CI) were used as the effect indicator for the dichotomous variables. $\mathrm{P}<0.05$ was considered to indicate a statistically significant difference between the two groups. Heterogeneity in all of the included studies was evaluated by $\chi^{2}$ and $I^{2}$ statistical tests. A random effects model was adopted when $\mathrm{P}<0.05$ or $I^{2}>50 \%$. Otherwise, the fixed-effect model was used. Taking into account the presence of non-randomized controlled trials (RCTs), a sensitivity analysis was performed to compare the incidence of PVST formation and anticoagulation-associated complications between patients receiving and not receiving prophylactic anticoagulation. A funnel plot was designed to establish the existence of publication bias.

\section{Results}

Search results and included studies. Overall, 1,099 potentially relevant articles were retrieved according to the search strategy. Among these, 118 were excluded due to duplication and 971 were excluded after reading the title and abstract. Thus, 10 studies were potentially eligible for this systematic review (Fig. 1). However, three studies were further excluded due to insufficient clinical data (lacking non- anticoagulation controls). Finally, seven studies were included in this review involving 383 and 283 patients with and without regular prophylactic anticoagulation, respectively (16-22) (Table I). Although two of the studies $(16,19)$ are from a single institution, they are not duplicates.

Of these seven studies, four were prospective, including one RCT, and three were retrospective studies. The years of publication spanned from 2006 to 2015. The included studies were conducted in four countries: Three in Japan, two in China, one in the Canada and one in Sweden. None of the seven studies was considered low quality and the study characteristics are presented in Table I.

Outcomes. All seven studies reported the incidence of PVST (16-22) (Table II). PVST was diagnosed by Doppler ultrasonography (US) in three studies and by Doppler US and contrast-enhanced $\mathrm{CT}$ in the other four studies. The fixed-effects model was adopted, as the heterogeneity analysis had not shown a significant difference. The incidence of PVST was found to be significantly reduced in the prophylactic anticoagulation group compared with the no prophylactic therapy/irregular anticoagulation group $(\mathrm{OR}=0.31,95 \% \mathrm{CI}$, 0.21-0.46; $\mathrm{P}<0.00001$ ) (Fig. 2). Due to heterogeneity in the study design, sensitivity analyses were conducted using the four prospective studies and the three retrospective studies. Evaluating the four prospective studies $(14,15,17,20)$, an anticoagulation effect was also found, with an OR of 0.21 (95\% CI, $0.08-0.52 ; \mathrm{P}=0.0008)$ and no heterogeneity $\left(\chi^{2}=4.53, \mathrm{P}=0.21\right.$, $\left.I^{2}=34 \%\right)$ with the fixed effect model. For the three retrospective studies $(18,20,21)$, heterogeneity was identified $\left(\chi^{2}=4.65\right.$, $\mathrm{P}=0.10, I^{2}=57 \%$ ), thus a random effects model was adopted and the anticoagulation effect was also found, with an OR of 0.34 (95\% CI, 0.14-0.82; $\mathrm{P}=0.02$ ).

Sensitivity analyses were performed according to the type of study population. In the subgroup analysis of two studies predominantly including the patients with hematological diseases $(17,20)$, the incidence of PVST was not significantly different between the prophylactic anticoagulation group and the control group with an OR of 3.27 (95\% CI, 0.36-29.57; $\mathrm{P}=0.29)$ and no heterogeneity $\left(\chi^{2}=0.00, \mathrm{P}=0.95, I^{2}=0 \%\right)$ 


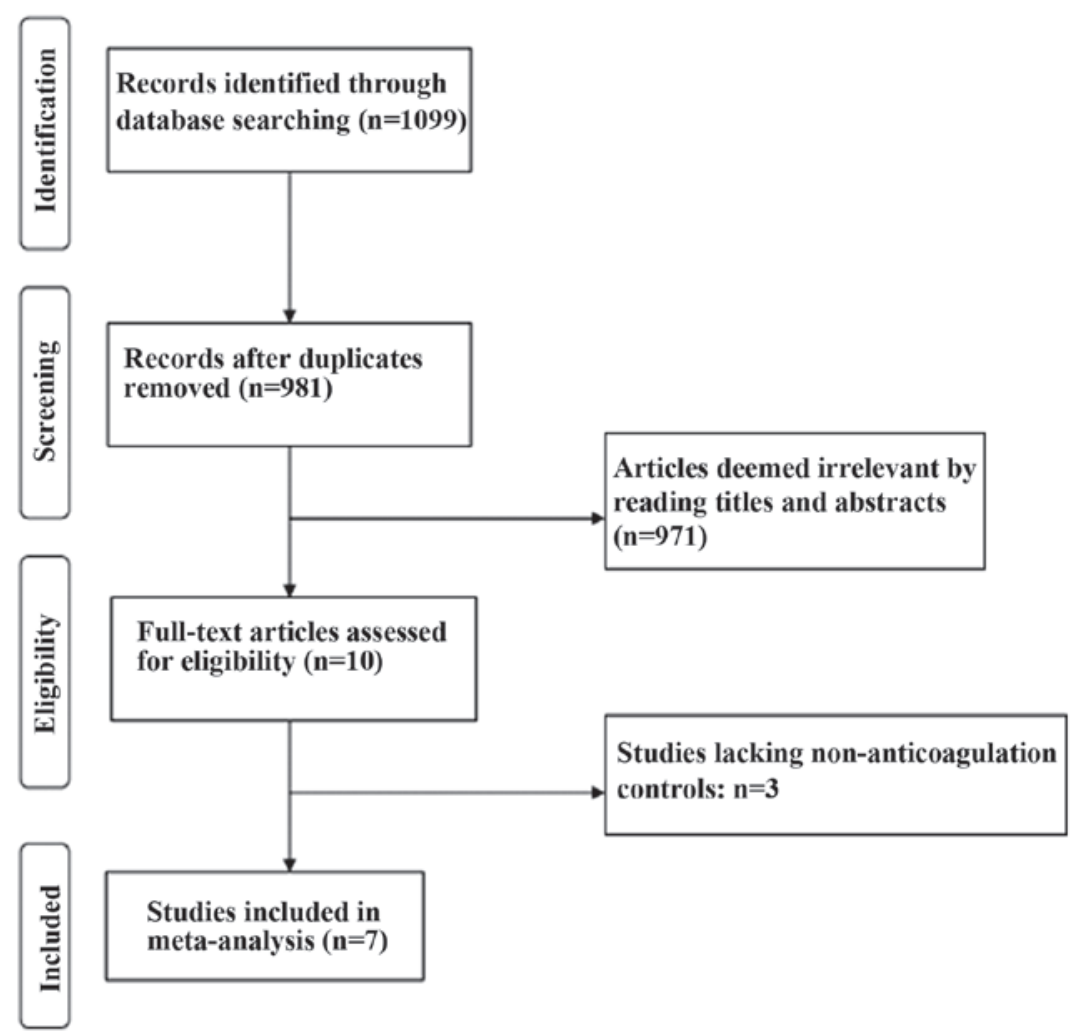

Figure 1. The preferred reporting items for systematic reviews and meta-analyses flow chart describing studies included in the meta-analysis.

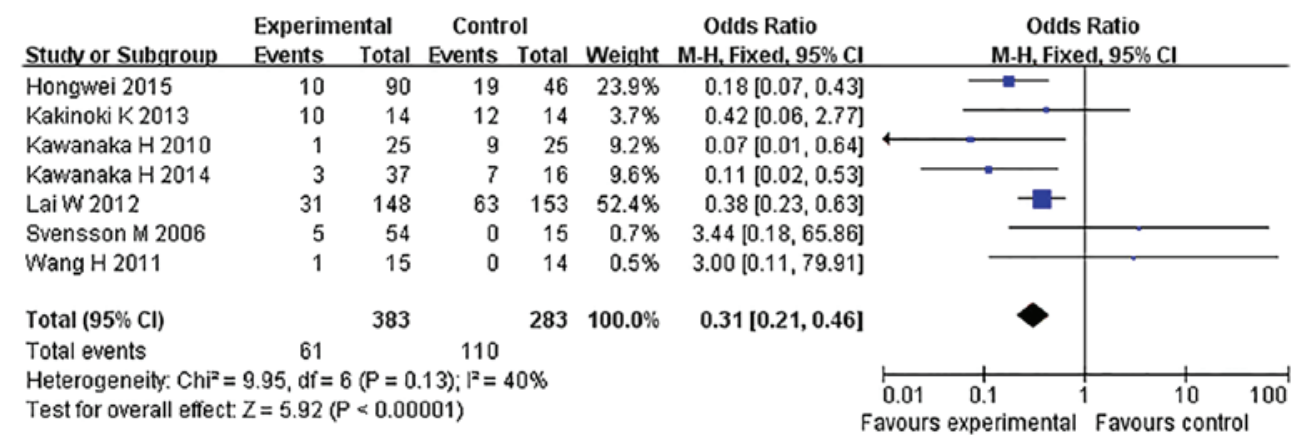

Figure 2. Portal vein system thrombosis rates for patients receiving vs. not receiving regular prophylactic anticoagulation in all studies. CI, confidence interval; M-H, Mantel-Haenszel test.

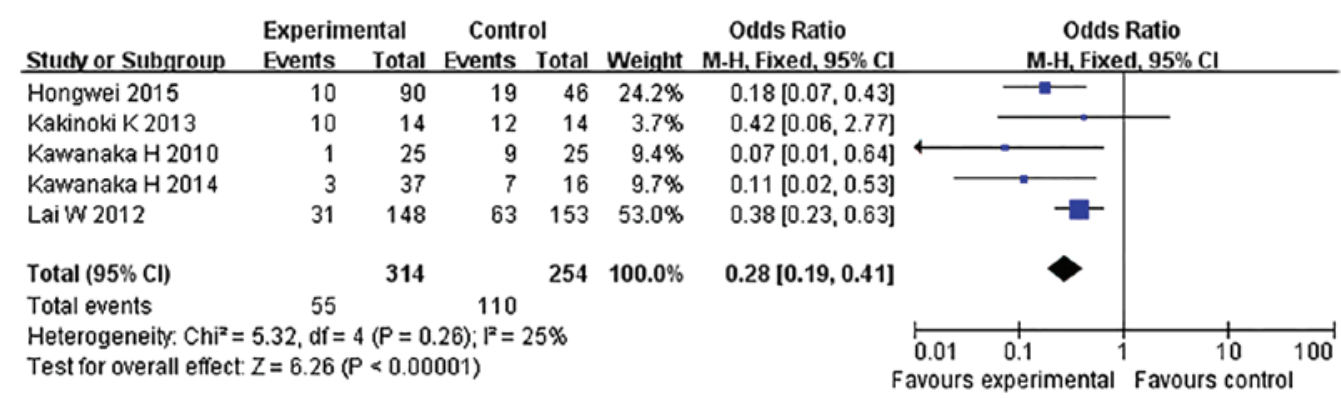

Figure 3. Portal vein system thrombosis rates for cirrhotic patients receiving vs. not receiving regular prophylactic anticoagulation. CI, confidence interval; M-H, Mantel-Haenszel test.

with the fixed-effect model. In the subgroup analysis of five studies including cirrhotic patients with hypersplenism, no heterogeneity was identified $\left(\chi^{2}=5.32, \mathrm{P}=0.26, I^{2}=25 \%\right)$, thus a fixed-effect model was adopted and an anticoagulation effect was identified, with an OR of 0.28 (95\% CI, 0.19-0.41; $\mathrm{P}<0.00001)$ (Fig. 3). 


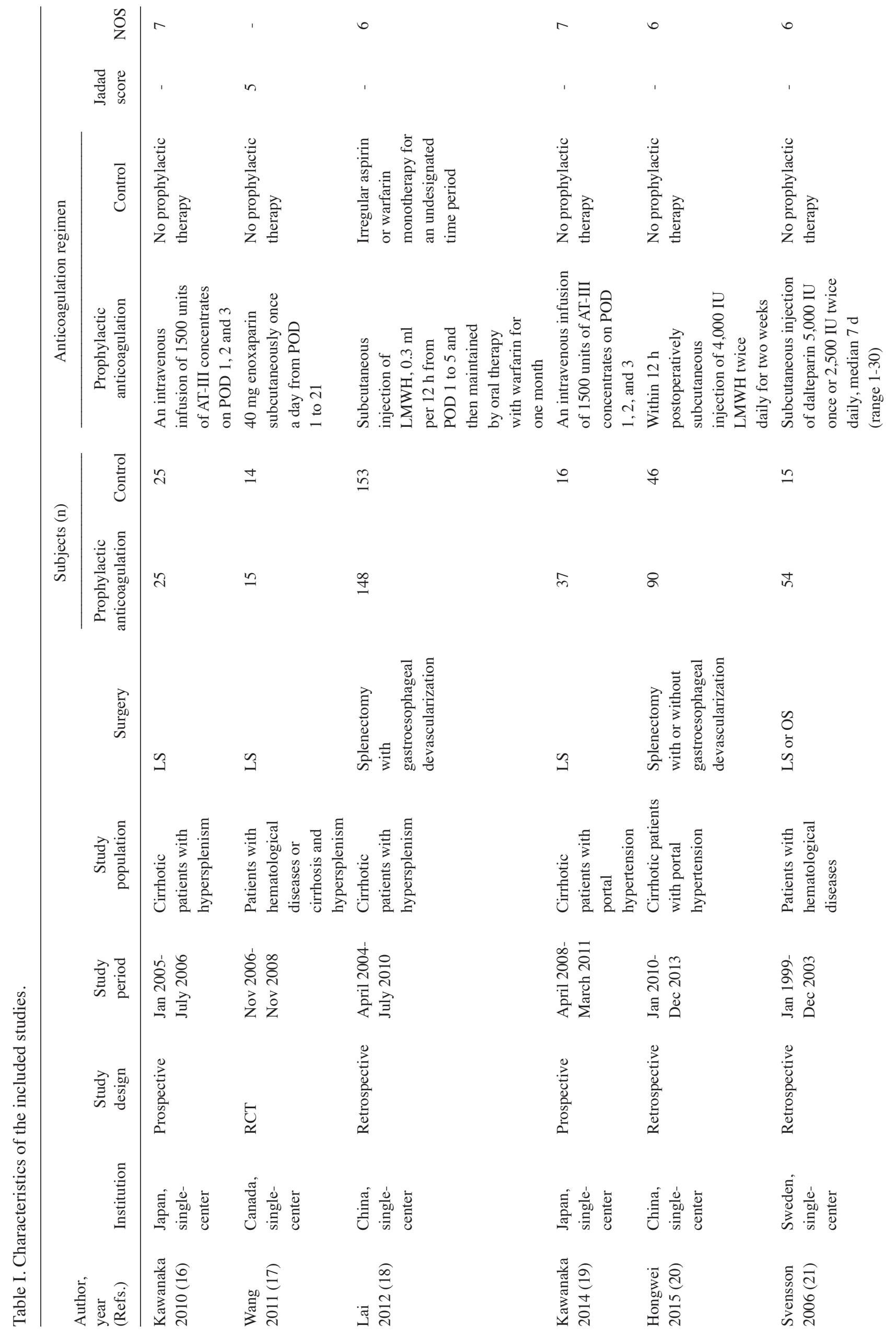


Among the seven studies, five provided the data regarding the incidence of anticoagulation-associated complications (Table II). During the anticoagulant therapy, complications were observed in three studies $(17,18,20)$, while no bleeding complications and hepatic damage were observed in the other two $(16,19)$. There was no heterogeneity $\left(\chi^{2}=1.60\right.$, $\left.\mathrm{P}=0.45, I^{2}=0 \%\right)$ and a fixed-effect model was therefore adopted. The meta-analysis showed that the incidence of anticoagulation-associated complications was not significantly different between the prophylactic anticoagulation group and the no prophylactic therapy/irregular anticoagulation group (OR=0.60, 95\% CI, 0.23-1.56; P=0.30) (Fig. 4). Due to heterogeneity in study design, sensitivity analyses were conducted. Again, the complication rates were not identified to be significantly different between the two groups using either prospective studies or retrospective studies (data not shown).

Publication bias. Given the limited number of studies $(n=7)$ in the current meta-analysis, no formal steps were taken, such as Egger's test, to determine publication bias.

\section{Discussion}

The reported incidence of PVST subsequent to splenectomy differs markedly, ranging from $0.36 \%$ (4) to $80 \%$ (23). There are various potential reasons for the inconsistency: i) The lack of typical symptoms in a large majority of PVST patients (24) results in diagnostic difficulties; ii) the detection rate of PVST differs depending on the examination methods (25); iii) the reported incidence of PVST varies greatly between different types of study. Previous studies, predominantly retrospective studies, may inaccurately report the incidence of PVST. By contrast, a number of prospective studies have reported a relatively higher incidence of PVST following splenectomy (24-26); iv) the incidence of PVST is also associated with the time and frequency of postoperative examinations; v) underlying diseases are also an important factor affecting the incidence. Patients with hematological diseases have relatively higher risk of PVST formation (25), whereas the risk is minimal for autoimmune disease and traumatic splenectomy $(26,27)$.

The detailed mechanisms for the formation of PVST following splenectomy remain unclear. However, it is generally hypothesized to be associated with local hypercoagulability occurring in the portal vein system subsequent to surgery, which may be attributed to soaring count and augmented aggregation competence of platelets post-surgery (28). Hemodynamic changes of the portal venous system may be another important reason for the formation of PVST. In the splenic vein stump induced by ligation (25), blood turbulence or stasis results in the deposition of blood cellular elements, which leads to the development of splenic vein thrombosis and subsequently, the development of portal and superior mesenteric vein thrombosis (2). In addition, operative manipulation, which may lead to serious damage of vascular endothelial cells and trigger the coagulation system (3), and irrational use of coagulants (29) also contributes to the development of PVST.

Although the prophylaxis of pulmonary embolism and deep vein thrombosis has been relatively well established, the prophylaxis of PVST following splenectomy remains 
Table II. Primary outcomes of the included studies.

\begin{tabular}{|c|c|c|c|c|}
\hline \multirow[b]{2}{*}{ Author, year (ref.) } & \multicolumn{2}{|c|}{ Patients developing PVST, n (\%) } & \multicolumn{2}{|c|}{$\begin{array}{l}\text { Patients developing anticoagulation-associated } \\
\text { complications, } \mathrm{n}(\%)\end{array}$} \\
\hline & Prophylactic anticoagulation & Control & Prophylactic anticoagulation & Control \\
\hline Kawanaka 2010 (16) & $1(4 \%)$ & $9(36 \%)$ & $0(0 \%)$ & $0(0 \%)$ \\
\hline Wang 2011 (17) & $1(6.67 \%)$ & $0(0 \%)$ & $1(6.67 \%)$ & $1(7.14 \%)$ \\
\hline Lai 2012 (18) & $31(20.94 \%)$ & $63(41.17 \%)$ & $2(1.35 \%)$ & $1(0.65 \%)$ \\
\hline Kawanaka 2014 (19) & $3(8.11 \%)$ & $7(43.75 \%)$ & $0(0 \%)$ & $0(0 \%)$ \\
\hline Hongwei 2015 (20) & $10(11.11 \%)$ & $19(41.30 \%)$ & $6(6.67 \%)$ & $7(15.22 \%)$ \\
\hline Svensson 2006 (21) & $5(9.26 \%)$ & $0(0 \%)$ & N/A & N/A \\
\hline Kakinoki 2013 (22) & $10(71.43 \%)$ & $12(85.71 \%)$ & N/A & N/A \\
\hline
\end{tabular}

PVST, portal vein system thrombosis; N/A, unavailable.

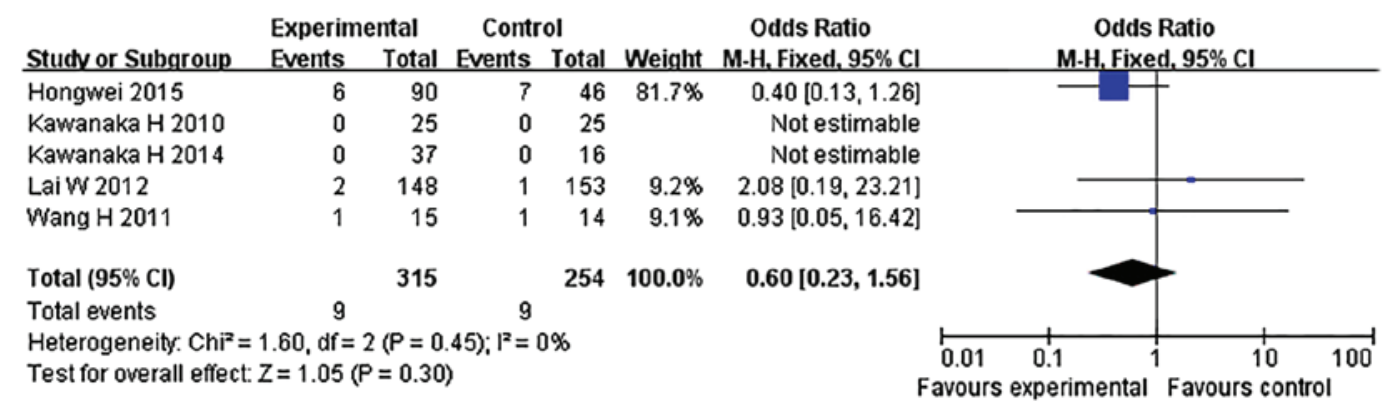

Figure 4. Complication rates for patients receiving vs. not receiving regular prophylactic anticoagulation in all studies. CI, confidence interval; $\mathrm{M}-\mathrm{H}$, Mantel-Haenszel test.

controversial. The primary concern is that prophylactic anticoagulation may induce the anticoagulation-associated complications, typically associated with bleeding. Currently, the management of PVST following splenectomy is predominantly based on individual experience (30) and a number of pilot studies demonstrated the feasibility, safety, and efficacy of prophylactic anticoagulation with decreased incidence of PVST and low rates of bleeding complications when compared with controls (16-20). However, due to the small number of cases in the individual studies, more results with adequate power are required to confirm these observations.

Recently, two meta-analyses regarding the role of anticoagulation in PVST have been performed $(31,32)$. However, one meta-analysis (31) focused on anticoagulation for the treatment of PVST, but not prevention of PVST. In the other meta-analysis (32), certain studies that were included may have been problematic: In the study by Ma et al (33), the authors compared different anticoagulation methods (radix Salviae miliorrhazae plus alprostadil vsersus radix Salviae miliorrhazae plus aspirin), instead comparing anticoagulation with non-anticoagulation. In the study by Xue et al (34), certain patients with high platelet counts in the control group also received anticoagulation. Therefore, it was considered necessary to perform the present meta-analysis comparing early prophylactic anticoagulation with no use of anticoagulation/irregular anticoagulation for the prevention of PVST following splenectomy, using the latest data.
According to the inclusion criteria, seven clinical studies were included in the present meta-analysis. In this meta-analysis, the incidence of PVST was identified to be significantly reduced in the prophylactic anticoagulation group when compared with a no prophylactic therapy/irregular anticoagulation group. In addition, the sensitivity analysis showed the same effect in the prospective and retrospective studies, respectively.

However, no significant difference was identified in the only RCT study (17). One explanation may be that this RCT is an underpowered study due to the small sample size. Notably, the study population in this RCT was predominantly patients with hematological diseases $(25 / 29 ; 86.21 \%)$. Therefore, sensitivity analyses were conducted according to the type of study population. The present results demonstrated that the use of anticoagulants did not affect the incidence of PVST subsequent to splenectomy in patients with hematological diseases. However, the implementation of prophylactic anticoagulation significantly reduced the incidence of PVST following splenectomy in cirrhotic patients with hypersplenis, which indicates that patients with hematological diseases may have a relatively lower risk of PVST formation compared with cirrhotic patients, and further multicenter clinical trials are required.

Another significant finding is that following surgery, the incidence of anticoagulation-associated complications was not significantly different between the prophylactic 
anticoagulation group and the no prophylactic therapy/irregular anticoagulation group. Wang et al (17) reported two patients, one in each group, experienced bleeding complications and the two patients were appropriately resuscitated. Anticoagulation was withheld temporarily in the patient in the anticoagulation group, and there were no long-term consequences. Lai et al (18) reported mild gastrointestinal bleeding in one patient in the anticoagulation group and in two in the control group. The anticoagulant therapy was terminated immediately and hemostatic agents were administered. Bleeding was successfully controlled and all patients recovered well. Hongwei et al (20) reported two cases of pancreatic leakage, three instances of subphrentic infection, and one case of surgical site bleeding in the anticoagulation group, whereas in the control group there were three, two and two cases, respectively. However, the authors did not describe methods to treat the complications. In the other four studies, no anticoagulation-associated complications were observed $(16,19)$ or there was no relevant data available $(21,22)$. These results strengthened the confidence that prophylactic anticoagulation may be safely administered subsequent to splenectomy, even for cirrhotic patients.

There are potential limitations of the current meta-analysis, which may increase the possibility of publication bias and affect the final result. First, this meta-analysis contained only seven studies and the number of cases was limited for this particular subject. In addition, only one RCT was included in this meta-analysis and the other non-RCT studies may have resulted in the unbalanced selection of patients. Furthermore, the study population, study design, surgical procedure, and anticoagulation regimens were variable and the heterogeneities were correlated with the habits and preferences of individual institutions. Finally, whether the selection of patients diagnosed with liver cirrhosis were treated or not treated with anticoagulant therapy was influenced by other factors, including severity of liver disease, esophageal varices and previous bleeding.

In conclusion, this meta-analysis demonstrates that early prophylactic anticoagulation is associated with a reduced incidence of PVST following splenectomy and is not associated with the incidence of anticoagulation-associated complications. However, due to the limitations of this analysis, further large multicenter RCTs are required to confirm this conclusion.

\section{References}

1. Parikh S, Shah R and Kapoor P: Portal vein thrombosis. Am J Med 123: 111-119, 2010.

2. Rattner DW, Ellman L and Warshaw AL: Portal vein thrombosis after elective splenectomy. An underappreciated, potentially lethal syndrome. Arch Surg 128: 565-570, 1993.

3. Stamou KM, Toutouzas KG, Kekis PB, Nakos S, Gafou A, Manouras A, Krespis E, Katsaragakis $\mathrm{S}$ and Bramis J: Prospective study of the incidence and risk factors of postsplenectomy thrombosis of the portal, mesenteric and splenic veins. Arch Surg 141: 663-669, 2006.

4. Delaitre B, Champault G, Barrat C, Gossot D, Bresler L, Meyer C, Collet D and Samama G: Laparoscopic splenectomy for hematologic diseases. Study of 275 cases. French society of laparoscopic surgery. Ann Chir 125: 522-529, 2000 (In French).

5. Mesa RA, Nagorney DS, Schwager S, Allred J and Tefferi A: Palliative goals, patient selection and perioperative platelet management: Outcomes and lessons from 3 decades of splenectomy for myelofibrosis with myeloid metaplasia at the Mayo Clinic. Cancer 107: 361-370, 2006.
6. van't Riet M, Burger JW, van Muiswinkel JM, Kazemier G, Schipperus MR and Bonjer HJ: Diagnosis and treatment of portal vein thrombosis following splenectomy. Br J Surg 87: 1229-1233, 2000.

7. Winslow ER, Brunt LM, Drebin JA, Soper NJ and Klingensmith ME: Portal vein thrombosis after splenectomy. Am J Surg 184: 631-636, 2002.

8. Kinjo N, Kawanaka H, Akahoshi T, Tomikawa M, Yamashita N, Konishi K, Tanoue K, Shirabe K, Hashizume M and Maehara Y: Risk factors for portal venous thrombosis after splenectomy in patients with cirrhosis and portal hypertension. Br J Surg 97: 910-916, 2010.

9. Northup PG and Intagliata NM: Anticoagulation in cirrhosis patients: What don't we know? Liver Int 31: 4-6, 2011.

10. Tripodi A, Primignani M, Chantarangkul V, Dell'Era A, Clerici M, de Franchis R, Colombo M and Mannucci PM: An imbalance of pro- vs anti-coagulation factors in plasma from patients with cirrhosis. Gastroenterology 137: 2105-2111, 2009.

11. Tripodi A and Mannucci PM: The coagulopathy of chronic liver disease. N Engl J Med 365: 147-156, 2011.

12. TripodiA:Thecoagulopathy of chronicliverdisease:Isthereacausal relationship with bleeding? No. Eur J Intern Med 21: 65-69, 2010.

13. Jadad AR, Moore RA, Carroll D, Jenkinson C, Reynolds DJ, Gavaghan DJ and McQuay HJ: Assessing the quality of reports of randomized clinical trials: Is blinding necessary? Control Clin Trials 17: 1-12, 1996.

14. Wells G, Shea B, O'Connell D, et al: The Newcastle-Ottawa Scale (NOS) for assessing the quality of nonrandomised studies in meta-analyses. http://www.ohri.ca/programs/clinical_epidemiology/oxford.asp. Accessed January 26, 2016.

15. Wang JZ, Liu Y, Wang JL, Lu L, Zhang YF, Lu HW and Li YM: Sequential vs simultaneous revascularization in patients undergoing liver transplantation: A meta-analysis. World J Gastroenterol 21: 7036-7046, 2015.

16. Kawanaka H, Akahoshi T, Kinjo N, Konishi K, Yoshida D, Anegawa G, Yamaguchi S, Uehara H, Hashimoto $\mathrm{N}$, Tsutsumi N, et al: Impact of antithrombin III concentrates on portal vein thrombosis after splenectomy in patients with liver cirrhosis and hypersplenism. Ann Surg 251: 76-83, 2010.

17. Wang H, Kopac D, Brisebois R, Sample C and Shapiro AM: Randomized controlled trial to investigate the impact of anticoagulation on the incidence of splenic or portal vein thrombosis after laparoscopic splenectomy. Can J Surg 54: 227-231, 2011.

18. Lai W, Lu SC, Li GY, Li CY, Wu JS, Guo QL, Wang ML and Li N: Anticoagulation therapy prevents portal-splenic vein thrombosis after splenectomy with gastroesophageal devascularization. World J Gastroenterol 18: 3443-3450, 2012.

19. Kawanaka H, Akahoshi T, Itoh S, Iguchi T, Harimoto N, Uchiyama H, Yoshizumi T, Shirabe K, Takenaka K and Maehara Y: Optimizing risk stratification in portal vein thrombosis after splenectomy and its primary prophylaxis with antithrombin III concentrates and danaparoid sodium in liver cirrhosis with portal hypertension. J Am Coll Surg 219: 865-874, 2014.

20. Hongwei C, Zhang L, Maoping L, Yong Z, Chengyou D and Dewei L: Era of liver transplantation: Combined anatomic splenectomy and anticoagulant therapy in prevention of portal vein thrombosis after splenectomy. Hepatogastroenterology 62: 405-409, 2015

21. Svensson M, Wirén M, Kimby E and Hägglund H: Portal vein thrombosis is a common complication following splenectomy in patients with malignant haematological diseases. Eur J Haematol 77: 203-209, 2006.

22. Kakinoki K, Okano K, Suto H, Oshima M, Hagiike M, Usuki H, Deguchi A, Masaki T and Suzuki Y: Hand-assisted laparoscopic splenectomy for thrombocytopenia in patients with cirrhosis. Surg Today 4: 883-888, 2013.

23. Romano F, Caprotti R, Conti M,Piacentini MG, Uggeri F, Motta V, Pogliani EM and Uggeri F: Thrombosis of the splenoportal axis after splenectomy. Langenbecks Arch Surg 391: 483-488, 2006.

24. Krauth MT, Lechner K, Neugebauer EA and Pabinger I: The postoperative splenic/portal vein thrombosis after splenectomy and its prevention-an unresolved issue. Haematologica 93: 1227-1232, 2008.

25. Ikeda M, Sekimoto M, Takiguchi S, Kubota M, Ikenaga M, Yamamoto H, Fujiwara Y, Ohue M, Yasuda T, Imamura H, et al: High incidence of thrombosis of the portal venous system after laparoscopic splenectomy: A prospective study with contrast-enhanced CT scan. Ann Surg 241: 208-216, 2005. 
26. Chaffanjon PC, Brichon PY, Ranchoup Y, Gressin R and Sotto JJ: Portal vein thrombosis following splenectomy for hematologic disease: Prospective study with Doppler color flow imaging. World J Surg 22: 1082-1086, 1998.

27. Bach AM, Hann LE, Brown KT, Getrajdman GI, Herman SK, Fong Y and Blumgart LH: Portal vein evaluation with US: Comparison to angiography combined with CT arterial portography. Radiology 201: 149-154, 1996.

28. Francoz C, Valla D and Durand F: Portal vein thrombosis, cirrhosis, and liver transplantation. J Hepatol 57: 203-212, 2012.

29. Soyer T, Ciftci AO, Tanyel FC, Senocak ME and Büyükpamukçu N: Portal vein thrombosis after splenectomy in pediatric hematologic disease: Risk factors, clinical features, and outcome. J Pediatr Surg 41: 1899-1902, 2006.

30. Li MX, Zhang XF, Liu ZW and Lv Y: Risk factors and clinical characteristics of portal vein thrombosis after splenectomy in patients with liver cirrhosis. Hepatobiliary Pancreat Dis Int 12: $512-519,2013$
31. Qi X, De Stefano V, Li H, Dai J, Guo X and Fan D: Anticoagulation for the treatment of portal vein thrombosis in liver cirrhosis: A systematic review and meta-analysis of observational studies. Eur J Intern Med 26: 23-29, 2015.

32. Qi X, Bai M, Guo X and Fan D: Pharmacologic prophylaxis of portal venous system thrombosis after splenectomy: A meta-analysis. Gastroenterol Res Pract 2014: 292689, 2014.

33. Ma JC, Zhao J, Su QH, Zhang DH, Guo Y and Ji ZZ: Effects of alprostadil in prevention of portal vein thrombogenesis after splenectomy and devascularization: A clinical observation of 76 patients with portal hypertension. Zhonghua Yi Xue Za Zhi 88: 524-526, 2008 (In Chinese).

34. Xue H, Zhang H, Zhang Y and Jiang Q: Portal anticoagulation in preventing thrombosis after porta-azygous devascularization for portal hypertension. Zhonghua Wai Ke Za Zhi 38: 855-857, 2000 (In Chinese). 